Original Research Paper

\title{
Enhanced Fatty Acid Production in Escherichia coli by Over- Expression of NADPH Generating Enzymes
}

\author{
${ }^{1}$ Huaiyuan Zhang, ${ }^{2}$ Yongshuang Zhou, ${ }^{2}$ Haiqin Chen, ${ }^{2}$ Yong Q. Chen and ${ }^{1}$ Yuanda Song \\ ${ }^{I}$ Colin Ratledge Center for Microbial Lipids, School of Agricultural Engineering and Food \\ Science, Shandong University of Technology, Zibo, 255000, Shandong, Republic of China \\ ${ }^{2}$ School of Food Science and Technology, Jiangnan University, Wuxi 214122, Jiangsu, Republic of China
}

Article history

Received: 19-07-2017

Revised: 01-09-2017

Accepted: 04-10-2017

Corresponding Author:

Yuanda Song

Colin Ratledge Center for

Microbial Lipids, School of

Agricultural Engineering and

Food Science, Shandong

University of Technology, Zibo,

255000, Shandong, People's

Republic of China

Emil: ysong@sdut.edu.cn

\begin{abstract}
NADPH is an essential factor for Fatty Acid (FA) biosynthesis. The effect of NADPH generating enzymes [NADP ${ }^{+}$-dependent malic enzyme (NADP-ME), $\mathrm{NADP}^{+}$-dependent isocitrate dehydrogenase (NADP-IDH), Glucose 6-Phosphate Dehydrogenase (GPD) and 6-Phosphogluconate Dehydrogenase (PGD)] on FA biosynthesis was investigated in an engineered Escherichia coli BL21 $\triangle$ fadE/pTE. Among NADPH-generating enzymes, GPD and ICD increased total FA production by 60 and $16 \%$ respectively. Especially medium-chain FA production was increased up to 4.2 and 2.5 folds respectively. In addition, overexpression of the endogenous Thioesterase (TE) reduced Cyclopropane Fatty Acid (CFA) production by $40 \%$ and over-expressing of GPD and ICD further decreased CFA production by 23 and 30\%, respectively compared to the TE overexpressed strain. Over-expression of TE appeared to be a good strategy to produce high quality biodiesel with medium-chain FAs as major Component (62\%) and with Minimal Amount of CFA (4\%). This study reveals that NADPHgenerating enzyme GPD is associated with FA biosynthesis in E. coli, but not all reducing power generating enzymes, are involved in FA biosynthesis in bacteria.
\end{abstract}

Keywords: Escherichia coli, Fatty Acid, Malic Enzyme, Reducing Power

\section{Introduction}

To meet the increasing fuel demand and to provide an alternative source to the limited fossil fuel supply, the development of renewable energy has become increasingly urgent. Biodiesel such as Fatty Acid Ethyl Ester (FAEE) and Fatty Acid Methyl Ester (FAME), which has high energy density and low water solubility (Bharathiraja et al., 2017), is rapidly moving toward the mainstream of alternative energy sources of fossil diesel. However, biodiesel is usually derived from animal fats or plant oils (triacylglycerides, TAGs), which is accompanied by high cost and inadequate supply. This then limited the development of biodiesel production. Microbial oil production by fermentation using a cheap carbon source offers a promising approach for the cost-effective production of biodiesel. Oleaginous microorganisms can accumulate a great deal of lipids, but slow growth rate and complicated regulation mechanisms of these organisms limit their use in biodiesel production (Meng et al., 2011). Escherichia coli has become an alternative Fatty Acid (FA) producer because it possesses several attractive advantages such as a known genomic sequence, available genetic tools and a rapid growth rate.

Nevertheless, in wild-type $E$. coli, both FA biosynthesis and degradation are regulated by transcriptional and post-transcriptional controls (Magnuson et al., 1993). In addition, its strong product feedback inhibition also works to maintain membrane lipid homeostasis (Fujita et al., 2007). Extensive genetic studies have been carried out to increase FA production in E. coli. So far, the major reported genetic strategies include four aspects: (1) expressing endogenous or exogenous thioesterase genes in E. coli to provide the cell with a free FA sink (Cho and Cronan, 1995; Steen et al., 2010), (2) blocking FA $\beta$-oxidation pathway by knocking out fadD or fadE (Duan et al., 2011; Lu et al., 2008; Steen et al., 2010), (3) enhancing the availability of the precursor malonyl-CoA by over expressing the AcetylCoA Carboxylase (ACC) (Davis et al., 2000; Lee et al., 2013) and (4) manipulation of the multi gene FA biosynthesis pathway and its global regulator (Xu et al., 2013; Zhang et al., 2012). However, only a few researches have been paid to the effect of NADPH generating enzymes on FA synthesis in $E$. coli, which provide the reducing power in the chain elongation pathway. 
The cofactor NADPH has generally been considered as exclusive reducing power for FA biosynthesis (Liu et al., 2010). There are several potential NADPH sources for FA biosynthesis (Fig. 1), such as NADP ${ }^{+}$ dependent malic enzyme (NADP-ME, EC 1.1.1.40), Glucose 6-Phosphate Dehydrogenase (GPD, EC 1.1.1.49), 6-phosphogluconate dehydrogenase (PGD, EC 1.1.1.44) and $\mathrm{NADP}^{+}$-dependent isocitrate dehydrogenase (NADPIDH, EC 1.1.1.42). The role of NADP-ME in filamentous fungi has been extensively studied (Ratledge and Wynn, 2002) and cytosolic NADP-ME appears to be the major NADPH provider for lipid accumulation. Also over-expression of NADP-ME leads to enhanced lipid accumulation in several fungi (Hao et al., 2014; Zhang et al., 2007). However, some oleaginous yeast such as Yarrowia lipolytica (Zhang et al., 2013a) and Lipomyces starkeyl (Tang et al., 2010) lack cytosolic NADP-ME, so most of the NADPH for FA biosynthesis is from Pentose Phosphate Pathway (PPP). Similarly, oleaginous microalga Chlorella protothecoides does not have a cytosolic NADP-ME activity and the PPP provided about $60 \%$ of NADPH for lipid biosynthesis, the rest of NADPH may be provided by NADP-ICDH (Xiong et al., 2010). Though, it is not clear whether this enzyme is in the cytoplasm or mitochondria. Cytosolic NADP-ICDH may be vital in FA biosynthesis when glucose is depleted from the culture (Varecza et al., 2006) or when the organism is grown on a non-fermentable carbon source (Minard and McAlister-Henn, 2005). However no clear evidence has been reported to link NADP-ICDH with FA biosynthesis in microorganisms, so far it is clear that NADP-ICDH plays a critical role in animal lipid metabolism (Koh et al., 2004).

The role of NADPH-generating enzymes on FA accumulation has barely been studied in bacteria. Questions such as, whether NADPH is a limiting factor for FA biosynthesis and whether some or all of the reducing power generating enzymes are involved in FA biosynthesis, remain elusive. It had reported in previous study (Meng et al., 2011) that over expression of NADP$\mathrm{ME}$ and adding malate to the medium significantly enhanced the FA yield in E. coli BL21, which is likely to be an artifact as the bacterium cannot accumulate FAs without a free FA metabolic sink. In this study, we overexpressed NADP-ME, NADP-IDH, GPD and PGD in an engineered $E$. coli strain BL21 $\Delta$ fadE/pTE with a FA metabolic sink and investigated the effects of these reducing power generating enzymes on FA production.

\section{Materials and Methods}

\section{Strains and Culture Medium}

E. coli TOP10 was used for the plasmid manipulations and E. coli BL21 (DE3) $\Delta$ fadE, donated by Lu et al. (2008), was used for protein expression. $E$. coli strains were grown in Luria Broth (LB) medium at $37^{\circ} \mathrm{C}$ during strains construction and M9 minimal medium (per liter: $20 \mathrm{~g}$ glucose, $6 \mathrm{~g} \mathrm{Na}_{2} \mathrm{HPO}_{4}, 1 \mathrm{~g}$ $\mathrm{NH}_{4} \mathrm{Cl}, \quad 0.5 \mathrm{~g} \mathrm{NaCl}$ and $0.24 \mathrm{~g} \mathrm{MgSO}_{4}$ ) for $\mathrm{FA}$ production. Antibiotics $\left(50 \mathrm{mg} \mathrm{L}^{-1}\right.$ kanamycin and 34 $\mathrm{mg} \mathrm{L}^{-1}$ chloramphenicol) were used for screening.

\section{Plasmids and Strains Construction}

Standard protocols were followed for the DNA manipulations (Sambrook and Russell, 2001). All of the primers in this study are showed in Table 1. Genomic DNA was isolated from E. coli BL21 (DE3) and the $m a e B$ gene encoding NADP-ME was amplified from genomic DNA using NADPME-F and NADPME-R primers, digested by Hind III and BamH I and cloned into pET28a $(+)$, resulting in pME. Genes icd, $z w f$ and gnd (encoding NADP-IDH, NADP-GPD and NADPPGD) were amplified from genomic DNA. PCR products were then cloned into pET28a $(+)$ resulting in pIDH, pGPD and pPGD plasmids (Table 2).

A pTE plasmid was also developed to provide $E$. coli with a FA metabolic sink by expressing a cytosolic thioesterase (TesA'). The tesA' gene with deleted signal peptide coding sequence was amplified from genomic DNA using the TE-F and TE-R primers and inserted behind the T7lac promoter of pACYCDuet-1 between BamH I and Hind III restriction enzyme cutting sites to obtain the pTE plasmid pTE was co-transformed with each of the plasmids (pET28a, pME, pIDH, pGPD and pPGD) into $E$. coli BL21 $\triangle$ fadE to construct the recombinant strains, designated YS1-YS5 (Table 2).

\section{Cultivation of E. coli Strains for FA Production}

E. coli strains were cultured in $300 \mathrm{ml} \mathrm{M9}$ medium (1 $\mathrm{L}$ flasks) with appropriate antibiotics at $37^{\circ} \mathrm{C}$. The induction of recombinant proteins was achieved by adding $0.5 \mathrm{mM}$ IPTG when the culture $\mathrm{OD}_{600}$ reached at 0.6. After induction, cell cultures were grown by shaking for $20 \mathrm{~h}$. Cells were then harvested by centrifugation $(6,000 \mathrm{~g}$ for $5 \mathrm{~min})$ and washed twice with distilled water for further analysis.

\section{Protein Determination and Enzyme Activity Assays in vitro}

The harvested cells were re-suspended with buffer a (Song et al., 2001) and disrupted by sonication. Cell debris was removed by centrifugation and the supernatant served as a crude extract. Protein concentration was determined by the Bradford method with BSA as a standard and the supernatant was also separated by Sodium Dodecyl Sulfate Polyacrylamide Gel Electrophoresis (SDS-PAGE).

The enzyme activity in vitro was determined spectrophotometrically by measuring the absorbance at 340 $\mathrm{nm}$ and $30^{\circ} \mathrm{C}$. The specific methods used were NADP-ME (Hsu and Lardy, 1969), NADP-IDH (Kornberg, 1955), GPD (Kuby and Noltmann, 1966) and PGD (Ruda et al., 2010). At least three measurements for each enzyme activity were carried out to assess reproducibility. 
Table 1. Primers used in this study

\begin{tabular}{llll}
\hline Genes & Primers & Sequences $(5 '-3$ ') & Annealing temperature $\left({ }^{\circ} \mathrm{C}\right)$ \\
\hline$m a e B$ & NADPME-F & CGCGGATCCGATGACCAGTTAAAACAAAG & 62.53 \\
\multirow{2}{*}{$i c d$} & NADPME-R & AGGCCCAAGCTTTTACAGCGGTTGGGTTTGCG & 69.76 \\
& IDH-F & CGCGGATCCGAAAGTAAAGTAGTTGTTCC & 61.91 \\
\multirow{2}{*}{$w f$} & IDH-R & AGGCCCAAGCTTTTACATGTTCTTGATGATCG & 63.08 \\
& GPD-F & CGCGGATCCGCGGTAACGCAAACAGCCCA & 71.89 \\
\multirow{2}{*}{ gnd } & GPD-R & AGGCCCAAGCTTTTACTCAAACTCATTCCAGG & 64.15 \\
& PGD-F & CGCGGATCCTCAAAGCAACAGATCGGCGTC & 68.43 \\
\multirow{2}{*}{$t e s A$} & PGD-R & AGGCCCAAGCTTTTAATCCAGCCATTCGGTATG & 65.68 \\
& TE-F & CGCGGATCCGGCGGACACGTTATTGATT & 66.95 \\
& TE-R & CCCAAGCTTTTATGAGTCATGATTTACT & 56.23 \\
\hline
\end{tabular}

The underlined portions indicate the restriction enzyme cutting sites

Table 2. Strains and plasmids used in this study

\begin{tabular}{|c|c|c|}
\hline Plasmids & Characteristics & Sources \\
\hline pET28a(+) & ColE1(pBR322), Kmr, T7lac promoter & Novagen \\
\hline pME & pET28a $(+)$ carrying $m a e B$ & This study \\
\hline pIDH & pET28a $(+)$ carrying $i c d$ & This study \\
\hline pGPD & pET28a $(+)$ carrying $z w f$ & This study \\
\hline pPGD & $\mathrm{pET} 28 \mathrm{a}(+)$ carrying gnd & This study \\
\hline pACYCDuet-1 & P15A(pACYC184), Camr, T7lac promoter & Novagen \\
\hline pTE & pACYCDuet-1 carrying 'tes $A$ & This study \\
\hline E. coli $\mathrm{BL} 21(\mathrm{DE} 3)$ & $\begin{array}{l}\text { F-omp T, hsdSB(rB-mB-), gal( } \lambda \text { cI857, ind1, Sam } 7 \\
\text { nin5, lacUV5-T7gene1), dcm(DE3) }\end{array}$ & Novagen \\
\hline E. coli TOP 10 & $\begin{array}{l}F-\text { mcrA } \Delta \text { (mrr-hsdRMS-mcrBC) } 980 \text { lacZ } \Delta \text { M15 } \Delta \text { lacX74 } \\
\text { recA1 ara } 139 \Delta \text { (ara-leu) } 7697 \text { galUgalKrpsL(Strr) endA1 nupG }\end{array}$ & Novagen \\
\hline E. coli BL21(DE3) $\triangle$ fadE & E. coli BL21 (DE3) lacking fadE & (Duan et al., 2011) \\
\hline YS1 & E. coli BL21 (DE3) $\Delta$ fadE/pTE/pET28a & This study \\
\hline YS2 & E. coli BL21 (DE3) $\Delta$ fadE/pTE/pME & This study \\
\hline YS3 & E. coli $\mathrm{BL} 21(\mathrm{DE} 3) \Delta \mathrm{fadE} / \mathrm{pTE} / \mathrm{pIDH}$ & This study \\
\hline YS4 & E. coli $\mathrm{BL} 21(\mathrm{DE} 3) \Delta \mathrm{fadE} / \mathrm{pTE} / \mathrm{pGPD}$ & This study \\
\hline YS5 & E. coli $\mathrm{BL} 21(\mathrm{DE} 3) \Delta \mathrm{fadE} / \mathrm{pTE} / \mathrm{pPGD}$ & This study \\
\hline
\end{tabular}

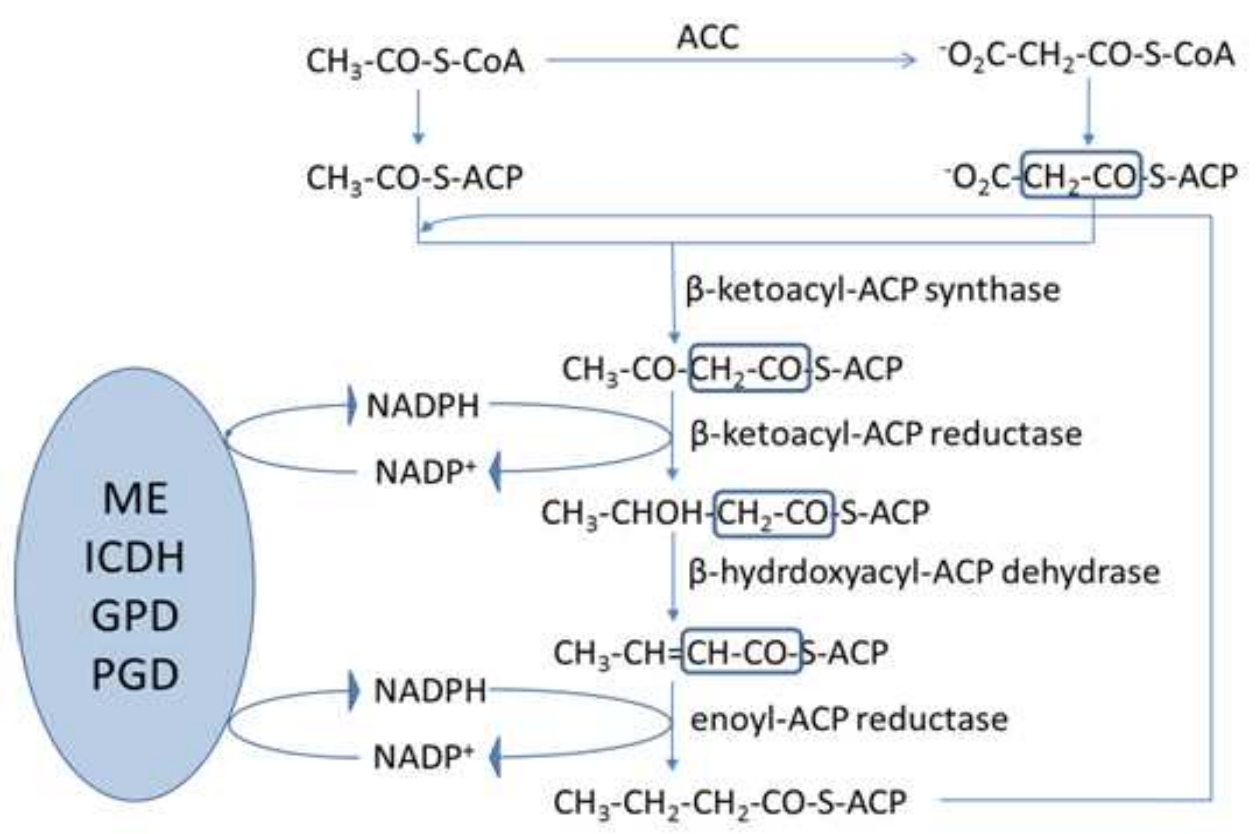

Fig. 1. FA biosynthesis pathway in E. coli. Reducing power NADPH, provided by ME, ICDH, GPD and PGD, is necessary for two reductive steps of FA biosynthesis pathway 


\section{Lipid isolation and FA analysis}

The harvested cells after freeze-drying $(100 \mathrm{mg})$ were subjected to lipids extraction by directly transmethylated according to Sakuradani and Shimizu (2003) with Pentadecanoic acid (C15:0, Sigma) as internal standard. FA Methyl Esters (FAMEs) were then analyzed by GC and GC/MS-MS according precious method (Hao et al., 2014).

\section{Results}

Recombinant Protein Expression in E. coli BL21 $\Delta f a d E$

To construct the strains with over expressed reducing power generating enzyme and a FA metabolic sink, the E. coli BL21 $\Delta$ fadE competent cells were co-transformed by pTE with each of the plasmids (pME, pIDH, pGPD and pPGD) designated YS2YS5 respectively (Table 2). The pET28a and pTE plasmids were co-transformed into E. coli BL21 $\Delta \mathrm{fadE}$ competent cells and the resulting strain was used as a control (YS1). The thioesterase (TesA') and reducing power generating enzymes were all induced by adding IPTG.

The expression level of these proteins was analyzed by SDS-PAGE (Fig. 2). All four proteins of NADP-ME, NADP-IDH, GPD and PGD were overproduced and the sizes of the induced proteins were in accordance with their estimated molecular weights 82, 46, 56 and $51 \mathrm{kDa}$, respectively (Fig. 2). A band of approximately $20 \mathrm{kDa}$ was present in all strains which was consistent with the estimated size of TesA' according to its gene sequence (Fig. 2).

The in vitro enzyme activities were also assayed and compared with the control strain YS1 (Table 3). Over-expression of NADP-ME, IDH, G6D and PGD in the recombinant strains led to about 826, 11, 96 and 14 folds increase in the corresponding enzyme activity, respectively.

\section{Over-Expression of NADPH Generating Enzymes Changed the FA Production}

To investigate the effects of over expressing enzymes on FA profile, we determined the FA yields of the reconstructed strains after IPTG induction for $20 \mathrm{~h}$ (Fig. 3). In the control strain YS1, the total FA production was $212.3 \mathrm{mg} / \mathrm{g}$ Dry Cell Weight (DCW). Among the other four strains, Over-expression of GPD in strain YS4 improved the FA yield by 1.6 fold. Strain YS3 (ICD over-expressing strain) showed a slight increase $(16 \%)$ in FA production, while YS5 strain had no significant difference with the control strain YS1. Surprisingly, over-expression of NADPME in strain YS2 reduced the FA production by $15 \%$ compared with the control strain.

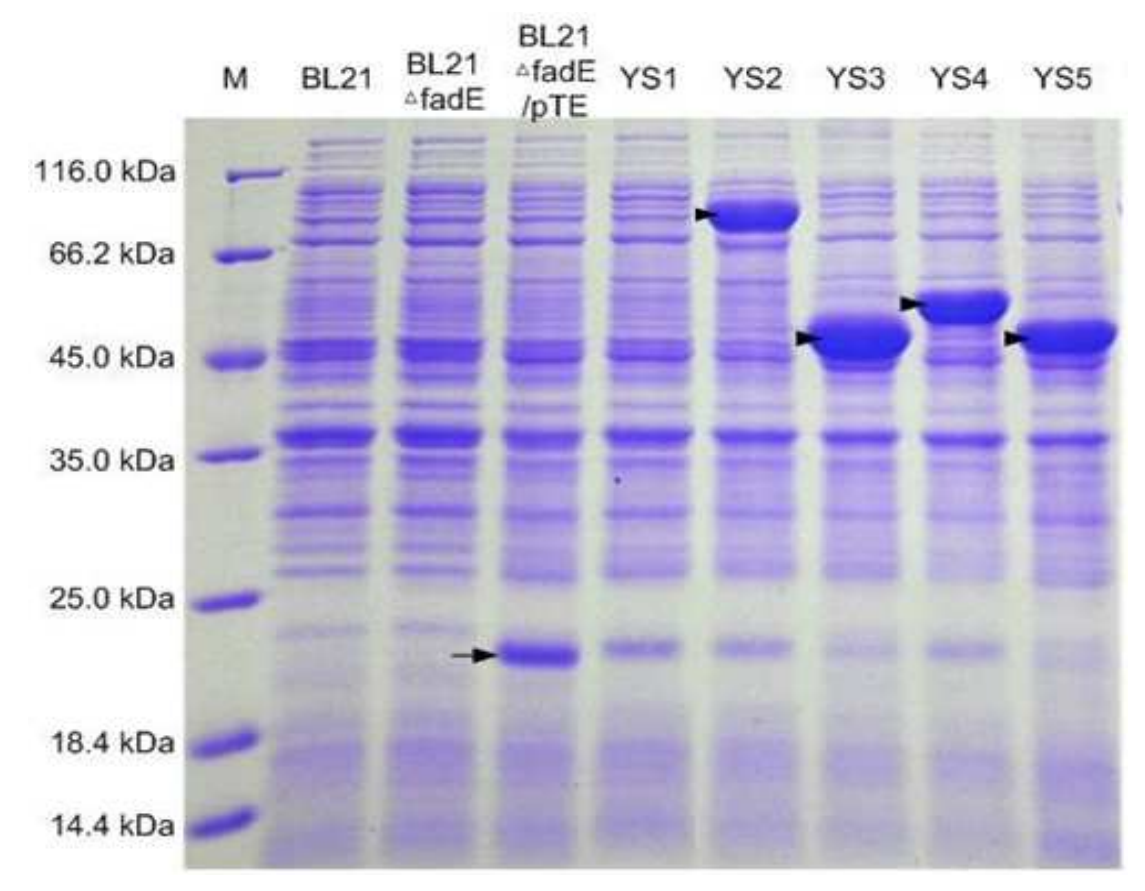

Fig. 2. SDS-PAGE analysis of overexpressed reducing power generating enzymes and 'TesA. The cells were cultivated in M9 culture medium for $20 \mathrm{~h}$ after induction and then harvested and broken for SDS-PAGE testing. The tags on the lanes stand for the different strains. The tailless arrows mark the overproduced reducing power generating enzymes and the normal arrow marks the 'TesA protein 


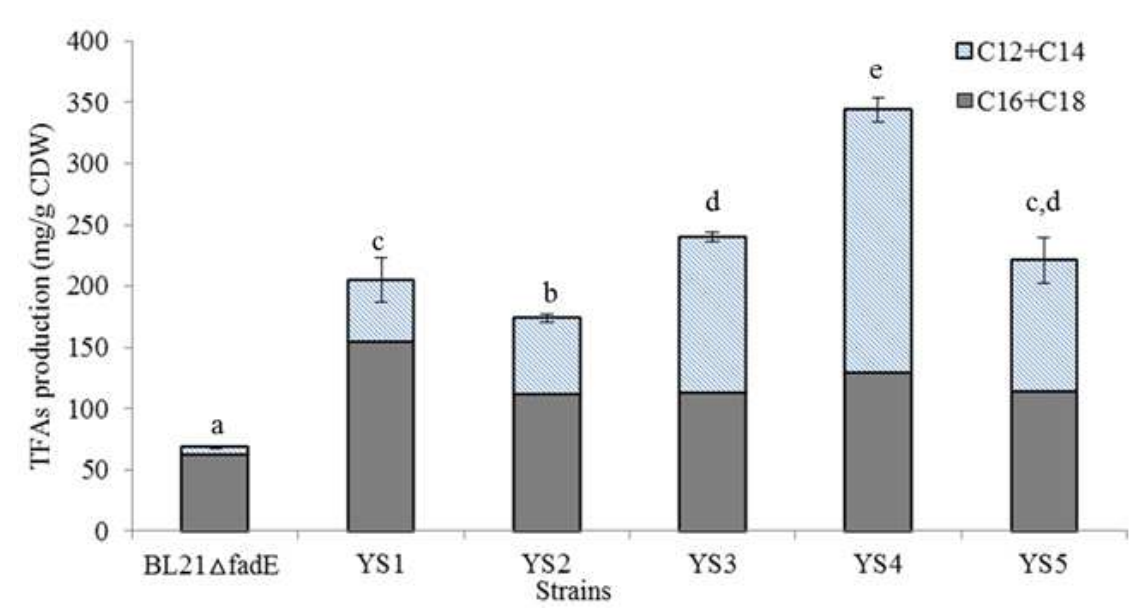

Fig. 3. Total Fatty Acids (TFAs) production in the control strain and five reducing power generating enzyme overexpressing strains. When $\mathrm{OD}_{600}$ reached $0.6,0.5 \mathrm{mM}$ IPTG was added to each strain culture. The cells were cultivated for $20 \mathrm{~h}$ after induction and harvested to analyze their FA production. The experiments were conducted in triplicate. The letters (a-e) on the columns denote significantly different (Duncan's multiple range test, $\mathrm{p}<0.05$ ) FA productions

Table 3. Enzyme activity

\begin{tabular}{lll} 
& & \\
& Enzyme activity $\left(\mathrm{nmol} \cdot \mathrm{min}^{-1} \cdot \mathrm{mg}_{\text {protein }}{ }^{-1}\right)$ & Over-expressing strain \\
Enzyme & $-\mathrm{-ontrol}$ strain & $9,915.6 \pm 231.5$ \\
NADP-ME & $12.5 \pm 0.8$ & $8,468.3 \pm 354.4$ \\
IDH & $750.6 \pm 23.3$ & $23,628.6 \pm 869.3$ \\
GPD & $247.2 \pm 16.1$ & $1,805.6 \pm 106.0$ \\
PGD & $127.1 \pm 2.6$ & \\
\hline
\end{tabular}

Data are expressed as the means \pm SD of the three replicates

Table 4. Fatty acid composition of each E. coli strain

\begin{tabular}{|c|c|c|c|c|c|c|c|c|c|c|c|}
\hline \multirow[b]{2}{*}{ Strains } & \multicolumn{11}{|c|}{ Fatty acid composition (w/w, \%) } \\
\hline & $\mathrm{C} 12: 0$ & $\mathrm{C} 12: 1$ & $\mathrm{C} 14: 0$ & $\mathrm{C} 14: 1$ & $\mathrm{C} 16: 0$ & $\mathrm{C} 16: 1$ & $\mathrm{C} 17: 0^{\Delta^{\mathrm{a}}}$ & $\mathrm{C} 18: 0$ & C18:1 & $\mathrm{C} 19: 0^{\Delta^{\mathrm{b}}}$ & UFA/SFAc \\
\hline BL21 $\Delta$ fadE & $2.52 \pm 0.04$ & N.D. & $6.98 \pm 0.02$ & $0.15 \pm 0.01$ & $44.52 \pm 0.06$ & $0.64 \pm 0.02$ & $25.48 \pm 0.12$ & $0.49 \pm 0.05$ & $1.11 \pm 0.01$ & $18.15 \pm 0.07$ & $0.02(0.84)$ \\
\hline YS1 & $24.35 \pm 0.60$ & $4.19 \pm 0.17$ & $35.13 \pm 0.37$ & $6.73 \pm 0.07$ & $8.08 \pm 0.20$ & $13.86 \pm 0.20$ & $1.66 \pm 0.40$ & $0.04 \pm 0.003$ & $5.63 \pm 0.06$ & $0.33 \pm 0.06$ & $0.45(0.48)$ \\
\hline YS2 & $7.08 \pm 0.44$ & $0.41 \pm 0.05$ & $26.47 \pm 0.68$ & $1.87 \pm 0.05$ & $25.98 \pm 0.39$ & $12.86 \pm 0.23$ & $6.20 \pm 1.33$ & $0.21 \pm 0.05$ & $16.50 \pm 0.55$ & $2.43 \pm 0.38$ & $0.46(0.67)$ \\
\hline YS3 & $13.41 \pm 0.11$ & $1.22 \pm 0.01$ & $33.75 \pm 0.43$ & $2.92 \pm 0.01$ & $18.85 \pm 0.25$ & $13.16 \pm 0.23$ & $4.01 \pm 0.90$ & $0.14 \pm 0.01$ & $11.33 \pm 0.36$ & $1.20 \pm 0.20$ & $0.40(0.51)$ \\
\hline YS4 & $18.77 \pm 0.30$ & $1.31 \pm 0.24$ & $37.52 \pm 1.02$ & $4.81 \pm 0.05$ & $12.23 \pm 0.39$ & $13.63 \pm 0.47$ & $2.90 \pm 0.70$ & $0.08 \pm 0.01$ & $7.55 \pm 0.27$ & $1.12 \pm 0.38$ & $0.38(0.46)$ \\
\hline YS5 & $12.56 \pm 0.20$ & $0.96 \pm 0.02$ & $32.40 \pm 0.41$ & $2.67 \pm 0.08$ & $20.62 \pm 0.90$ & $12.50 \pm 0.32$ & $4.57 \pm 0.90$ & $0.15 \pm 0.01$ & $11.87 \pm 0.59$ & $1.71 \pm 0.25$ & $0.39(0.52)$ \\
\hline
\end{tabular}

a, C17:0 $0^{\Delta}$, cis-9,10-methylenehexadecanoic acid. b, C19:0 , cis-9,10-methyleneoctadecanoic acid. c, the ratio of total Unsaturated FA (UFA) to Saturated FA (SFA), the No. in the bracket showed the ratio when C17:0 and C19:0 cyclopropane FAs were treated as unsaturated FAs. N.D.= not detectable

\section{Over-Expression of NADPH Generating Enzymes Changed the FA Composition}

The FA compositions of the reconstructed strains were also analyzed by GC-MS and were shown in Table 4. In the fadE-knockout strain, C16:0 was the most abundant FAs (44.52\%), followed by two CFA, cis-9,10methylenehexadecanoic acid $\left(\mathrm{C} 17: 0^{\Delta}\right)$ and cis-9,10methyleneoctadecanoic acid $\left(\mathrm{C} 19: 0^{\Delta}\right)$. Over-expression of thioesterase (control strain YS1) reduced the production of $\mathrm{C} 17: 0^{\Delta}$ and $\mathrm{C} 19: 0^{\Delta}$ greatly and as a result, increased $\mathrm{C} 16: 1$ and $\mathrm{C} 18: 1$ concentration, furthermore it also increased medium chain FAs (C12-C14) production. Over-expression of reducing power generating enzymes in TE genetic background leads to the further decrease of CFA production and massive increase of the medium FA production, especially GPD increased the medium FA production for about $300 \%$. However the overall ratio of unsaturated FAs verses saturated FAs (UFA/SFA) was slightly decreased. The FA profile of NADP-ME over-expressing strain, which decreased the total FA production slightly, was similar to the control strain YS1.

\section{Discussion}

Genetic engineering of $E$. coli for the production of FAs or biodiesel has been studied extensively in the past decade and the yield of FA production in E. coli has been increased up to $8.6 \mathrm{~g} \mathrm{~L}^{-1}$ (Xu et al., 2013), however, lipid content per cell dry weight is only about $21 \%$, there is still much room to be improved. The main 
genetic work has focused on reconstructing the metabolic pathway for FA production but not much works had been done to investigate the effect of NADPH generating enzymes on FA production. Although one report showed that over-expression of NADP-ME increased FA production in E. coli BL21 significantly (Meng et al., 2011), but this result was irreproducible in our study. We conducted a preliminary experiment with E. coli BL21 (DE3) as a host strain to over express NADP-ME and other reducing power generating enzymes to compare the FA production. In comparison with the control strain E. coli BL21/pET28a, the FA production of the over-expressing strains did not increase but rather presented modest decreases. Although the corresponding substrate of the enzymes was added to the culture medium, the FA production stayed the same as that of the cells without exogenous substrates (data not shown). In fact, genetically non-engineered $E$. coli does not accumulate FAs and FA metabolic pathways must be regulated tightly to maintain membrane lipid homeostasis. So what Meng et al. (2011) found is very likely an artifact. Liu et al. (2010) also suggested that under the physiological condition of E. coli XL100 lacking the $f a d D$ gene, the NADPH supply is unlikely to be the limiting factor in FA production. Therefore, only when $E$. coli was engineered to have a FA metabolic sink by genetic modifications such as over-expressing a TesA', then its FA production capacity can be manipulated by over-expression of NADPH-generating enzymes or other enzymes that regulate FA production.

In oleaginous eukaryotic microorganisms, only cytosolic NADPH-generating enzymes, such as ME, GPD and PGD, are involved in FA biosynthesis. However in prokaryotic microorganisms, as mitochondrion is absent, all NADPH-generating enzymes may be involved in anabolic pathways. In our work, among the NADPH-generating enzymes, GPD of PPP has most effectively increased the FA production $(60 \%)$, ICD of TCA cycle slightly increased the FA production $(16 \%)$. These results are consistent with the findings regarding genetic engineering of NADPH regenerators for enhanced production of GDP-L-fucose in $E$. coli as over-expression of GPD and ICD increased GDP-L-fucose production by $46,31 \%$, respectively (Lee et al., 2011). Also these findings appear to be true for other non-FA production pathways that need NADPH as reviewed by (Lennen et al., 2010). Solid evidences of the contributions of individual NADPH generating enzymes for anabolic metabolism has been found by metabolic flux analysis. Earlier work did by Sauer et al. (2004) showed that PPP, ICD and transhydrogenase provided 35-35, 20-25 and 35-45\% NADPH respectively in E. coli MG 1655. A previous work conducted by He et al. (2014) showed that PPP, ICD and transhydrogenase provided 32,8 and $60 \%$ NADPH respectively for biosynthetic pathway in a FA-over-production engineered strain (E. coli DH1).

Although it has generally been assumed that NADPME provides NADPH for the biosynthesis of FA in various eukaryotic microorganisms (Ratledge and Wynn, 2002), over expression of NADP-ME did not lead to increased FA production in this study. On the contrary, it decreased FA production for $15 \%$. Similarly, a previous work showed that over-expression of NADP-ME in $E$. coli BL21 reduced GDP-L-fucose production by $24 \%$ metabolic flux analysis by He et al. (2014) has clearly showed that flux ratio through NADP-ME is negligible in both control and engineered $E$. coli, this suggests that NADP-ME is not involved in providing NADPH for FA biosynthesis. Potential role of NADP-ME has been suggested to provide NADPH for bacterial growth on two carbon compounds, such as acetate (Wang et al., 2011). Therefore, the extremely high expression of a "non-functional" NADP-ME in our experiment (the in vitro NADP-ME activity in strain YS2 was 826 times that in the control strain YS1) either burdened the host or caused an imbalanced metabolism in the FA pathway (Yu et al., 2011), which lead to the decreased production of FA.

Similar to previous reports (Cao et al., 2010; Voelker and Davies, 1994), a large amount of CFA was produced in fadE-knockout strain of E. coli BL21 cultivated for $20 \mathrm{~h}$, which is commonly produced by methylenation of the unsaturated FA of membrane phospholipid in bacteria grown at late exponential to stationary phase. CFA inhibits the activity of glycerol 3phosphate acyltransferase (Kito et al., 1972) and works as a double switch to control phospholipid biosynthesis at stationary phase when cell proliferation is arrested, which resulted in the accumulation of acyl-ACPs and leads to the inhibition of FA synthesis. However, when an endogenous Thioesterase (TE) was expressed in the cytoplasm of $E$. coli, the synthesized acyl-ACPs can be hydrolyzed to release free FAs and thus feed-back inhibition on FAS was removed, which lead to the great increase of FA production (about 200\% increase). Furthermore, CFA production was greatly inhibited (40\% decrease), while unsaturated $\mathrm{C} 16: 1$ and $\mathrm{C} 18: 1$ production was massively increased ( 55 fold) in TE over expressing E. coli. Similar results has been reported by Voelker and Davies (1994) who observed that CFA (C17:0) is greatly reduced, while unsaturated FAs in phopholipid fraction is largely increased in E. coli strain over expressing a plant acyl-ACP thioesterase. This is likely due to the redirection of acyl-ACPS flux to free FA synthesis and limited the production of phospholipids, the substrate for CFA production. However if we consider CFAs as unsaturated FAs (as CFAs are produced from membrane phopholipid carried unsaturated FAs), the ratio of UFA/SFA was even slightly decreased rather than increased. 
The effect of TEs on FA production and FA composition has been extensively studied in $E$. coli (Fan et al., 2013; Jing et al., 2011; Zhang et al., 2013b) and medium chain acyl-ACP TEs appear to be efficient for the production of medium chain FAs. In this study, over-expression of endogenous TE in E. coli increased the production of medium-chain FAs (C12-C14) and long chain FAs $(\mathrm{C} 16-\mathrm{C} 18)$ by 8 and 2 folds respectively. This is not in accordance with the nature of endogenous TE of E. coli, as it prefers long chain FAs (C16-C18) to medium-chain FAs (C12-14) as substrates (Spencer et al., 1978). This is likely due to the fact that TE hydrolyze the intermediate product of long chain acyl-ACPs and released the feed-back inhibition of ketoacyl-ACP synthase III and enoyl-ACP reductase in the elongation cycle (initiation) of FA biosynthesis (Richard and Rock, 1996). This lead to the activated initiation of FA synthesis, resulted in a deficiency of malonyl-CoA for the terminal elongation step, thus more medium-chain FAs were produced.

Over-expression of GPD in TE-over expressed $E$. coli, resulted in massive increase of medium chain FAs (3.2 fold increase), while the long chain FAs (C16-C18) production is slightly decreased (Fig. 3). This suggests that overproduced NADPH, a substrate for enoyl-ACP reductase, further activate FA biosynthetic pathway, especially the initiation step, which leads to a deficiency of malonyl-CoA for the terminal elongation step (Richard and Rock, 1996; Magnuson et al., 1993). Therefore long chain FA production is restricted and more medium chain FA production is increased. In addition, CFA production was further reduced by $23 \%$ in G8PD over expressed strains respectively. Thus, engineering $E$. coli by co-expression of TE and NADPHgenerating enzymes can provide a good cell factory to produce FAs with medium chain FAs as major component (62\%, of TFA) and minimal amount of CFA (4\%, of TFA), which is excellent source for biodiesel production.

Free FAs have been detected in the culture medium in $E$. coli with expressing the TesA' enzyme (Davis et al., 2000). However, we did not detect any FA in the culture medium (cells were excluded), suggesting that overproduced FAs might be intracellular, which is not too high to be secreted to the culture medium. To maximize the FA yields of these strains, culture medium and cultivation conditions are necessary to be optimized.

\section{Conclusion}

Four strains were constructed with over expressed four reducing power generating enzymes in E. coli BL21 $\triangle \mathrm{fadE} / \mathrm{pTE}$. Among the NADPH generating enzyme GPD of PPP increased FA production $(60 \%)$ more than ICD of TCA cycle (16\%). Over-expression of endogenous TE in E. coli, not only increased the total
FA production, but also increased unsaturated FA content and medium-chain FA (C12-C14) production. In addition, it greatly decreased CFA production at stationary phase. Over-expressing of NADPHgenerating enzymes, especially GPD decreased CFA production. More importantly, it extraordinarily increased the production of medium-chain FAs, possibly because of activation of FA initiation by its synergistic action with TE.

\section{Acknowledgement}

This work was supported by the National Natural Science Foundation of China (No. 31271812 and 31670064), the National High Technology Research and Development Program of China (863 Program No. 2012AA022105C), Taishan Industry Leading Talent Project and starting grant from Shandong University of Technology. Furthermore we would like to thank Dr. Xuefeng Lu for strain gift.

\section{Author's Contributions}

Huaiyuan Zhang: Participated in all experiments, coordinated the data-analysis and contributed to the writing of the manuscript.

Yongshuang Zhou: Participated in all experiments, coordinated the data-analysis and contributed to the writing of the manuscript.

Haiqin Chen: Designed the research plan, organized the study.

Yong Q. Chen: Designed the research plan, organized the study.

Yuanda Song: Designed the research plan, organized the study and amended the manuscript.

\section{Ethics}

This article is original and contains unpublished material. The corresponding author confirms that all of the other authors have read and approved the manuscript and no ethical issues involved.

\section{References}

Bharathiraja, B, S. Sridharan, V. Sowmya, D. Yuvaraj and R. Praveenkumar, 2017. Microbial oil - A plausible alternate resource for food and fuel application. Bioresour Technol., 233: 423-432.

Cao, Y., J. Yang, M. Xian, X. Xu and W. Liu, 2010. Increasing unsaturated fatty acid contents in Escherichia coli by coexpression of three different genes. Applied Microbiol. Biotechnol., 87: 271-280. DOI: $10.1007 / \mathrm{s} 00253-009-2377-\mathrm{x}$

Cho, H. and J.E. Cronan, 1995. Defective export of a periplasmic enzyme disrupts regulation of fatty acid synthesis. J. Biol. Chem., 270: 4216-4219. 
Davis, M.S., J. Solbiati and J.E. Cronan, 2000. Overproduction of acetyl-CoA carboxylase activity increases the rate of fatty acid biosynthesis in Escherichia coli. J. Biol. Chem., 275: 28593-28598.

Duan, Y., Z. Zhu, K. Cai, X. Tan and X. Lu, 2011. De novo biosynthesis of biodiesel by Escherichia coli in optimized fed-batch cultivation. PLoS One. DOI: 10.1371/journal.pone.0020265

Fan, L., J. Liu, K. Nie, L. Liu and F. Wang et al., 2013. Synthesis of medium chain length fatty acid ethyl esters in engineered Escherichia coli using endogenously produced medium chain fatty acids. Enzyme Microb. Technol., 53: 128-33.

Fujita, Y., H. Matsuoka and K. Hirooka, 2007. Regulation of fatty acid metabolism in bacteria. Mol. Microbiol., 66: 829-839.

Hao, G.H., H.Q. Chen, L. Wang, Z.N. Gu and Y.D. Song et al., 2014. Role of Malic Enzyme during fatty acid synthesis in the oleaginous fungus Mortierella alpina. Applied Environ. Microbiol., 80: 2672-2678.

He, L., Y. Xiao, N. Gebreselassie, F. Zhang and M.R. Antoniewiez et al., 2014. Central metabolic responses to the overproduction of fatty acids in Escherichia coli based on 13C-metabolic flux analysis. Biotechnol. Bioeng., 111: 575-585.

Richard, J.H. and C.O. Rock, 1996. Regulation of fatty acid elongation and initiation by acyl-acyl carrier protein in Escherichia coli. J. Biol. Chem., 271: 1833-1836.

Hsu, R.Y. and H.A. Lardy, 1969. Malic enzyme. Methods Enzymol., 13: 230-235.

Jing, F., D.C. Cantu, J. Tvaruzkova, J.P. Chipman and Nikolau, B.J. Yandeau-Nelson et al., 2011. Phylogenetic and experimental characterization of an acyl-ACP thioesterase family reveals significant diversity in enzymatic specificity and activity. BMC Biochem., 12: 44-44. DOI: 10.1186/1471-2091-12-44

Kito, M., S. Aibara, K. Hasegawa and T. Hata, 1972. Inhibition of L-glycerol 3-phosphate acyltransferase from Escherichia coli by cis-9, 10methylenehexadecanoic acid. J. Biochem., 71: 99-105.

Koh, H.J., S.M. Lee, B.G. Son, S.H. Lee and Z.Y. Ryoo et al., 2004. Cytosolic NADP+-dependent isocitrate dehydrogenase plays a key role in lipid metabolism. J. Biol. Chem., 279: 39968-39974.

Kornberg, A., 1955. Isocitric dehydrogenase of yeast. Methods Enzymol., 1: 705-709.

Kuby, S.A. and E.A. Noltmann, 1966. Glucose 6phosphate dehydrogenase (crystalline) from brewers' yeast. Methods Enzymol., 9: 116-125.

Lee, W.H., Y.W. Chin, N.S. Han, M.D. Kim and J.H. Seo, 2011. Enhanced production of GDP-L-fucose by overexpression of NADPH regenerator in recombinant Escherichia coli. Applied Microbiol. Biotechnol., 91: 967-976. DOI: 10.1007/s00253-011-3271-x
Lee, W.H., M.D. Kim, Y.S. Jin and J.H. Seo, 2013. Engineering of NADPH regenerators in Escherichia coli for enhanced biotransformation. Applied Microbiol. Biotechnol., 97: 2761-72.

Lennen, R.M., D.J. Braden, R.A. West, J.A. Dumesic and B.F. Pfleger, 2010. A process for microbial hydrocarbon synthesis: Overproduction of fatty acids in Escherichia coli and catalytic conversion to alkanes. Biotechnol. Bioeng., 106: 193-202.

Liu, T., H. Vora and C. Khosla, 2010. Quantitative analysis and engineering of fatty acid biosynthesis in E. coli. Metab. Eng., 12: 378-386.

Lu, X., H. Vora and C. Khosla, 2008. Overproduction of free fatty acids in E. coli: Implications for biodiesel production. Metab. Eng., 10: 333-339.

Magnuson, K., S. Jackowski, C.O. Rock and J.E. Cronan, 1993. Regulation of fatty acid biosynthesis in Escherichia coli. Microbiol. Rev., 57: 522-542.

Meng, X., J. Yang, Y. Cao, L. Li and X. Jiang et al., 2011. Increasing fatty acid production in E. coli by simulating the lipid accumulation of oleaginous microorganisms. J. Ind. Microbiol. Biotechnol., 38: 919-925. DOI: $10.1007 / \mathrm{s} 10295-010-0861-\mathrm{z}$

Minard, K.I. and L. McAlister-Henn, 2005. Sources of NADPH in yeast vary with carbon source. J. Biol. Chem., 280: 39890-39896.

Varecza, Z., T. Emri, T. Pusztahelyi and I. Pócsi, 2006. A novel aspect of NADPH production in ageing Penicillium chrysogenum. Acta. Biol. Hung., 57: 115-121. DOI: 10.1556/ABiol.57.2006.1.11

Voelker, T.A. and H.M. Davies, 1994. Alteration of the specificity and regulation of fatty acid synthesis of Escherichia coli by expression of a plant mediumchain acyl-acyl carrier protein thioesterase. J. Bacteriol., 176: 7320-7327.

Wang, B., P. Wang, E. Zheng, X. Chen and H. Zhao et al., 2011. Biochemical properties and physiological roles of NADP-dependent malic enzyme in Escherichia coli. J. Microbiol., 49: 797-802.

Ratledge, C. and J.P. Wynn, 2002. The biochemistry and molecular biology of lipid accumulation in oleaginous microorganisms. Adv. Applied Microbiol., 51: 1-51.

Ruda, G.F., G. Campbell, V.P. Alibu, M.P. Barrett and R. Brenk et al., 2010. Virtual fragment screening for novel inhibitors of 6-phosphogluconate dehydrogenase. Bioorg. Med. Chem., 18: 5056-5062.

Sakuradani, E. and S. Shimizu, 2003. Gene cloning and functional analysis of a second $\Delta 6$-fatty acid desaturase from an arachidonic acid-producing Mortierella fungus. Biosci. Biotech Bioch., 67: 112-120. DOI: 10.1271/bbb.67.704

Sambrook, J. and D.W. Russell, 2001. Molecular Cloning: A Laboratory Manual. 1st Edn., CSHL Press,, Cold Spring Harbor, ISBN-10: 0879695773, pp: 2344. 
Sauer, U., F. Canonaco, S. Heri, A. Perrenoud and E. Fischer, 2004. The soluble and membrane-bound transhydrogenases UdhA and PntAB have divergent functions in NADPH metabolism of Escherichia coli. J. Biol. Chem., 279: 6613-6619.

Song, Y.D., J.P. Wynn, Y. Li, D. Grantham and C. Ratledge, 2001. A pre-genetic study of the isoforms of malic enzyme associated with lipid accumulation in Mucor circinelloides. Microbiology, 147: 1507-1515.

Spencer, A.K., A.D. Greenspan and J.E. Cronan, 1978. Thioesterases I and II of Escherichia coli. Hydrolysis of native acyl-acyl carrier protein thioesters. J. Biol. Chem., 253: 5922-5926.

Steen, E.J., Y. Kang, G. Bokinsky, Z. Hu and A. Schirmer et al., 2010. Microbial production of fattyacid-derived fuels and chemicals from plant biomass. Nature, 463: 559-562.

Tang, W., S. Zhang, H. Tan and Z.K. Zhao, 2010. Molecular cloning and characterization of a malic enzyme gene from the oleaginous yeast Lipomyces starkeyi. Mol. Biotechnol., 45: 121-128.

Xiong, W., L.X. Liu, C. Wu, C. Yang and Q.Y. Wu, 2010. 13C-tracer and gas chromatography-mass spectrometry analyses reveal metabolic flux distribution in the oleaginous microalga Chlorella protothecoides. Plant Physiol., 154: 1001-1011.
Xu, P., Q. Gu, W. Wang, L. Wong and A.G. Bower et al., 2013. Modular optimization of multi-gene pathways for fatty acids production in E. coli. Nat. Commun., 4: 1409-1409. DOI: $10.1038 /$ ncomms 2425

Yu, X., T. Liu, F. Zhu and C. Khosla, 2011. In vitro reconstitution and steady-state analysis of the fatty acid synthase from Escherichia coli. Proc. Natl. Acad. Sci., 108: 18643-18648.

Zhang, F., M. Ouellet, T.S. Batth, P.D. Adams and C.J. Petzold et al., 2012. Enhancing fatty acid production by the expression of the regulatory transcription factor FadR. Metab. Eng., 14: 653-660.

Zhang, H.Y., L.N. Zhang, H.Q. Chen, Y.Q. Chen and C. Ratledge et al., 2013a. Regulatory properties of malic enzyme in the oleaginous yeast, Yarrowia lipolytica and its non-involvement in lipid accumulation. Biotech Lett., 35: 2091-2098.

Zhang, X., M. Li, A. Agrawal and K.Y. San, 2013b. Efficient free fatty acid production in Escherichia coli using plant acyl-ACP thioesterases. Metab. Eng., 13: 713-722.

Zhang, Y., I.P. Adams and C. Ratledge, 2007. Malic enzyme: the controlling activity for lipid production? Over expression of malic enzyme in Mucor circinelloides leads to a 2.5-fold increase in lipid accumulation. Microbiology, 153: 2013-2025. 